\title{
Optimization for Lattices and Diophantine Approximations
}

\author{
Masaru ITO $^{1}$ and Noriko HIRATA-KOHNO ${ }^{2, *, \dagger}$ \\ ${ }^{1}$ Department of Mathematical and Computing Sciences, \\ Tokyo Institute of Technology, Meguro, Tokyo 152-8552, Japan \\ ${ }^{2}$ Department of Mathematics, College of Science and Technology, \\ Nihon University, Chiyoda, Tokyo 101-8308, Japan
}

Received January 16, 2013; final version accepted May 14, 2013

\begin{abstract}
In this article, we optimize a growth condition such that an analytic function being almost integer-valued at integers turns out to be a polynomial. Our argument relies on a computational optimization combined with Diophantine approximations on lattices.
\end{abstract}

KEYWORDS: lattice, almost integer-valued function, optimization, Diophantine approximation

\section{Introduction}

Denote by $\mathbb{N}$ the set of strictly positive rational integers. Let us investigate the nature of an entire function $F(z)$ in one complex variable such that $F(\mathbb{N}) \subset \mathbb{Z}$, or $F(z)$ taking values close to integers at any $z \in \mathbb{N}$.

Our natural questions which arise are as follows.

(1) Is $F(z)$ a polynomial?

The answer is no, because of the existence of the functions such that $F(z)=2^{z}$ or $3^{z}$, etc. Then the second question arises:

(2) Does there exist a suitable condition such that $F(z)$ becomes a polynomial?

Denote by $\overline{\mathbb{Q}}$ the algebraic closure of $\mathbb{Q}$ in $\mathbb{C}$. Consider a complex function $F(z)$ in $z \in \mathbb{C}$ which satisfies $F(u) \in \overline{\mathbb{Q}}$ for any $u \in \overline{\mathbb{Q}}$. We may now ask the following more general question:

(3) Is $F(z)$ a polynomial or an algebraic function?

Indeed, there exist examples due to P. Stäckel [9] in 1895 of transcendental functions taking algebraic values at all algebraic points. Hence the answer to the third question is again, no, namely it is true that such $F(z)$ supposed to verify $F(u) \in \overline{\mathbb{Q}}$ for any $u \in \overline{\mathbb{Q}}$, is not necessarily algebraic function.

On the other hand, the Hermite-Lindemann theorem in transcendental number theory shows $\exp (\alpha) \notin \overline{\mathbb{Q}}$ for any $\alpha \in \overline{\mathbb{Q}}$, $\alpha \neq 0$, which says, there exists a transcendental function always taking transcendental values at any non-trivial algebraic point. Moreover, the Gel'fond-Schneider theorem notices $2^{\sqrt{3}} \notin \overline{\mathbb{Q}}$, then even an entire function $F(z)=2^{z}$ with $F(\mathbb{N}) \subset \mathbb{Z}$ does not satisfy $F(\overline{\mathbb{Q}}) \subset \overline{\mathbb{Q}}$.

Now let us restrict ourselves to consider an entire function $F(z)$ such that $F(\mathbb{N}) \subset \mathbb{Z}$. For our question (2), we refer to a fundamental result of G. Pólya [8] concerning with such functions.

Recall the definition of the order of a complex function. Definition 1. Let $F(z)$ be an entire function in $\mathbb{C}$. Write $|F|_{r}=\sup _{|z| \leq r}|F(z)|$ and define $\alpha(F)$ the order of exponential
type of $F(z)$ by

$$
\alpha(F)=\limsup _{r \rightarrow+\infty} \frac{\log |F|_{r}}{r}
$$

Theorem A (Pólya). Let $F(z)$ be an entire function in $\mathbb{C}$ with $F(\mathbb{N}) \subset \mathbb{Z}$. Suppose $\alpha(F)<\log 2$. Then $F(z)$ is a polynomial.

Pólya also observed the case $F(\mathbb{Z}) \subset \mathbb{Z}$ as follows.

Theorem B (Pólya). Let $F(z)$ be an entire function in $\mathbb{C}$ with $F(\mathbb{Z}) \subset \mathbb{Z}$. Suppose $\alpha(F)<\log \left(\frac{3+\sqrt{5}}{2}\right)$. Then $F(z)$
is a polynomial.

2010 Mathematics Subject Classification: 11C08, 11K60, 11Y70, 30D15.

*Corresponding author. E-mail: hirata@math.cst.nihon-u.ac.jp

The second author is supported by the NEXT Program, GR 087, JSPS for 2010-2013. 
We see that $F(z)$ in theorems of Pólya is necessarily a polynomial in $\mathbb{Q}[z]$, but not in $\mathbb{Z}[z]$ [consider for example, $\left.\frac{1}{2} z(z+1)\right]$. The bounds of the order $\log 2$ and $\log \left(\frac{3+\sqrt{5}}{2}\right)$ are optimal because of the existence of the functions $2^{z}$ and $\left(\frac{3+\sqrt{5}}{2}\right)^{z}+\left(\frac{3-\sqrt{5}}{2}\right)^{z}$.

It is equivalent to say that $F(z)$ is a polynomial with coefficients in $\mathbb{Q}$, and that the functions $z^{h} F(z)^{k}(h, k \in \mathbb{N} \cup\{0\})$ are linearly dependent over $\mathbb{Q}$. Then a natural generalization of works of Pólya is to seek a sufficient condition such that several functions, say, $f_{1}(z), \cdots, f_{L}(z)$, are linearly dependent over $\mathbb{Q}$.

For $\zeta_{1}, \zeta_{2}, \cdots \in \mathbb{C}$ denote by $r(N):=\max _{1 \leq n \leq N}\left|\zeta_{n}\right|$. We proved in [5]:

Theorem $\mathbf{C}([5])$. Let $L$ and $N_{0}$ be integers with $1<N_{0}<L$. There are constants $C_{1}>0$ and $C_{2}>0$ depending only on $L, N_{0}$ satisfying the following. Let $\zeta_{1}, \zeta_{2}, \cdots \in \mathbb{C}$ be infinitely many pairwise distinct complex points. Let $f_{1}(z), \cdots, f_{L}(z)$ be entire functions in $\mathbb{C}$. Suppose $f_{j}\left(\zeta_{n}\right) \in \mathbb{Z}$ for any $j, n$ with $1 \leq j \leq L$ and $n \geq 1$. If we have $\max _{1 \leq j \leq L} \log \left|f_{j}\right|_{C_{1} r(N)} \leq C_{2} N$ for any $N \geq N_{0}$, then the functions $f_{1}(z), \cdots, f_{L}(z)$ are linearly dependent over $\mathbb{Q}$.

A consequence of Theorem $\mathrm{C}$ is for instance, a weaker version of Theorem $\mathrm{A}$ :

Corollary D ([5]). Let $F(z)$ be an entire function in $\mathbb{C}$ with $F(\mathbb{N}) \subset \mathbb{Z}$. Suppose $\alpha(F) \leq \frac{1}{40}$. Then $F(z)$ is a polynomial over $\mathbb{Q}$.

Proof. Consider $\frac{z(z-1) \cdots(z-h+1)}{h !} \cdot F(z)^{k}$ as $f_{1}(z), \cdots, f_{L}(z)$ in Theorem $\mathrm{C}(h, k \in \mathbb{N} \cup\{0\})$.

\section{Results for an Almost Integer-Valued Function}

Our aim in the present article is to relax the hypothesis of theorem of Pólya from the viewpoint of Diophantine approximations.

Definition 2. We write $\|z\|:=\min _{m \in \mathbb{Z}}|z-m|$, the distance between $z \in \mathbb{C}$ and the nearest integer.

In this section, by means of computational optimization joined with Diophantine approximation method, we prove the following results. These theorems show that an almost integer-valued entire function is a polynomial over $\mathbb{Q}$, provided that the growth of the function is sufficiently bounded.

Theorem 1. Let $f(z)$ be an entire function in $\mathbb{C}$ with $\alpha(f) \leq 0.00437155$. Suppose

$$
\|f(n)\|<e^{-663143 n}
$$

for all sufficiently large $n \in \mathbb{N}$. Then $f(z) \in \mathbb{Q}[z]$.

A slight modification of the proof enables us to obtain:

Theorem 2. Let $f(z)$ be an entire function in $\mathbb{C}$ with $\alpha(f) \leq 8.775 \times 10^{-6}$. Suppose

$$
\|f(n)\|<e^{-3 n}
$$

for all sufficiently large $n \in \mathbb{N}$. Then $f(z) \in \mathbb{Q}[z]$.

We note $0.00437155>\frac{1}{229}$ and $8.775 \times 10^{-6}>\frac{1}{113961}$.

Theorems 1 and 2 show, not only an integer-valued entire function but also an almost integer-valued function, namely a function taking values very close to integers, may turn out to be a polynomial. This is an improvement of a result in [5] concerning with a growth condition or an assumption for the distance from the nearest integer. Theorems 1 and 2 give explicit versions of a theorem due to $\mathrm{Ch}$. Pisot dealing with an almost integer-valued function (Théorème 2 in [7]). We prove both theorems by so-called Schneider's method (refer its applications found in [3, 10]) of Diophantine approximations, that differs from Pisot's original proof.

\section{Computational Lemmata}

We collect here a few classical estimates. Throughout the article, we denote $\varphi(x)=x \log x$. Lemma 3. Let $N_{0}$ be a sufficiently large integer. Let $h_{0} \in \mathbb{Q}$ such that $h_{0} N_{0} \in \mathbb{Z}, 0<h_{0}<\frac{1}{2}$. Let $n, h$ be integers
with $0 \leq n \leq N_{0}-1,1 \leq h \leq h_{0} N_{0}$. Then we have

$$
\log \left|\left(\begin{array}{l}
n \\
h
\end{array}\right)\right| \leq-N_{0}\left\{\varphi\left(h_{0}\right)+\varphi\left(1-h_{0}\right)\right\}+\mathrm{o}\left(N_{0}\right) .
$$

Proof. Since $h_{0}<\frac{1}{2}$, we have 


$$
\left|\left(\begin{array}{l}
n \\
h
\end{array}\right)\right| \leq\left(\begin{array}{c}
N_{0} \\
h
\end{array}\right) \leq\left(\begin{array}{c}
N_{0} \\
h_{0} N_{0}
\end{array}\right)
$$
thus the Stirling formula (see chapter XII, §33, page 251 of [12]) for $\frac{N_{0} \text { ! }}{\left(N_{0}\left(1-h_{0}\right)\right) ! \cdot\left(h_{0} N_{0}\right) !}$ gives the required
estimate.

Indeed this is a reason why we use binomial polynomials instead of usual monomials as a basis of ring of polynomials.

Lemma 4. Let $\lambda>1$ be a parameter $\in \mathbb{R}$ and $N \geq 2$ be an integer. Put $\Lambda=\lambda^{2 \lambda^{2}-1}\left(\lambda^{2}-1\right)^{1-\lambda^{2}}>1$. There exists a constant $C$ with $\frac{1}{2}<C<4$ satisfying the following.

Let $F(z)$ be an entire function in $\mathbb{C}$ with $F(n)=0$ for all $n=0,1, \cdots, N-1$. Then we have

$$
\log |F(N)| \leq \log |F|_{\lambda N}-N \log \Lambda+C \log N .
$$

If $N \geq 10^{5}$ then we have a more precised estimate:

$$
\log |F(N)| \leq \log |F|_{\lambda N}-N \log \Lambda+\frac{1}{2} \log (N+1)+\frac{1}{2} \log \frac{2 \pi\left(\lambda^{2}-1\right)}{\lambda^{2}}+\theta
$$

with

$$
-\frac{73}{36}<\theta<\frac{10}{9}+\frac{1}{2} \log \frac{\lambda^{2}}{\lambda^{2}-1}
$$

Proof. We put

$$
Q(z)=Q_{(N)}(z)=\prod_{n=0}^{N-1} \frac{\lambda N(z-n)}{\lambda^{2} N^{2}-z n} .
$$

The function $Q$ and $\frac{F}{Q}$ are analytic in the disk $|z| \leq \lambda N$.

Since $|Q(z)|=1$, we have for $|z|=\lambda N$ :

$$
\left|\frac{F(N)}{Q(N)}\right| \leq\left|\frac{F}{Q}\right|_{\lambda N} \leq|F|_{\lambda N}
$$

Therefore we obtain $|F(N)| \leq Q(N)|F|_{\lambda N}$.

On the other hand, by an asymptotic expansion of the logarithm of the Gamma function (called Stirling's series, see [12]), we have for $x>0$,

$$
\Gamma(x)=x^{x-\frac{1}{2}} e^{-x}(2 \pi)^{\frac{1}{2}} e^{\frac{\theta_{0}}{12 x}}
$$

with certain $\theta_{0}, 0<\theta_{0}<1$.

It is well-known that Euler's formula implies for $z$ which is not negative integer (chapter XII, §12, page 237 of [12]):

$$
\Gamma(z+1)=z \Gamma(z)
$$

consequently we have

$$
\Gamma\left(\lambda^{2} N+1\right)=\lambda^{2} N\left(\lambda^{2} N-1\right) \cdots\left(\lambda^{2} N-N+1\right) \cdot \Gamma\left(\lambda^{2} N-N+1\right)
$$

from which we get

$$
\prod_{n=0}^{N-1}\left(\lambda^{2} N-n\right)=\frac{\Gamma\left(\lambda^{2} N+1\right)}{\Gamma\left(\lambda^{2} N-N+1\right)} .
$$

Hence we obtain

$$
\begin{aligned}
|Q(N)| & =\frac{(\lambda N)^{N} \cdot \Gamma(N+1) \cdot \Gamma\left(\lambda^{2} N-N+1\right)}{N^{N} \cdot \Gamma\left(\lambda^{2} N+1\right)} \\
& =\frac{\sqrt{2 \pi} \cdot \lambda^{N} \cdot(N+1)^{N+\frac{1}{2}} \cdot\left(\lambda^{2} N-N+1\right)^{\lambda^{2} N-N+\frac{1}{2}} \cdot e^{\frac{\theta_{1}}{12(N+1)}} e^{\frac{\theta_{2}}{12\left(\lambda^{2} N-N+1\right)}}}{\left(\lambda^{2} N+1\right)^{\lambda^{2} N+\frac{1}{2}} \cdot e \cdot e^{\frac{\theta_{3}}{12\left(\lambda^{2} N+1\right)}}}
\end{aligned}
$$

with $0<\theta_{i}<1(1 \leq i \leq 3)$.

Thanks to a simple estimate for $x>0$ :

$$
(x+1)^{x}=x^{x} \cdot e^{a}
$$

with certain $0<a<1$, we have 


$$
\begin{aligned}
\log |Q(N)|= & -N \log \Lambda+\frac{1}{2} \log (N+1)+\frac{1}{2} \log \frac{\lambda^{2} N-N+1}{\lambda^{2} N+1} \\
& +\frac{1}{2} \log 2 \pi-1+a_{1}+a_{2}-a_{3} \\
& +\frac{\theta_{1}}{12(N+1)}+\frac{\theta_{2}}{12\left(\lambda^{2} N-N+1\right)}-\frac{\theta_{3}}{12\left(\lambda^{2} N+1\right)} \\
= & -N \log \Lambda+\frac{1}{2} \log (N+1)+\frac{1}{2} \log \left(2 \pi\left(1-\frac{1}{\lambda^{2}}\right)\right)+\theta
\end{aligned}
$$

where $0<a_{i}<1(1 \leq i \leq 3), 0<\theta_{i}<1(1 \leq i \leq 3),-\frac{73}{36}<\theta<\frac{10}{9}+\frac{1}{2} \log \frac{\lambda^{2}}{\lambda^{2}-1}$.

This completes the proof.

Lemma 5. Let $f(z)$ be an analytic function on a disk $|z| \leq R$ in $\mathbb{C}$. Let $\zeta_{0}, \ldots, \zeta_{\ell}$ be pairwise distinct points in $|z|<R$. Then we have

$$
\left|f\left(\zeta_{0}\right)\right| \leq E_{1}+E_{2}
$$

where

$$
\begin{aligned}
& E_{1}=|f|_{R} \cdot \frac{R}{R-\left|\zeta_{0}\right|} \prod_{n=1}^{\ell} \frac{\left|\zeta_{0}-\zeta_{n}\right|}{R-\left|\zeta_{n}\right|}, \\
& E_{2}=\prod_{k=1}^{\ell}\left|\zeta_{0}-\zeta_{k}\right| \cdot \sum_{n=1}^{\ell}\left(\frac{\left|f\left(\zeta_{n}\right)\right|}{\left|\zeta_{0}-\zeta_{n}\right|} \cdot \prod_{\substack{i=1 \\
i \neq n}}^{\ell} \frac{1}{\left|\zeta_{i}-\zeta_{n}\right|}\right) .
\end{aligned}
$$

Proof. Put

$$
A(z)=\prod_{n=1}^{\ell} \frac{R-\zeta_{n}}{z-\zeta_{n}}
$$

and for $1 \leq n \leq \ell$ write also

$$
A_{n}(z)=A(z) \cdot\left(z-\zeta_{n}\right)=\prod_{n=1}^{\ell}\left(R-\zeta_{n}\right) \cdot \prod_{\substack{i=1 \\ i \neq n}}^{\ell} \frac{1}{z-\zeta_{i}}
$$

The residue formula gives

$$
A\left(\zeta_{0}\right) f\left(\zeta_{0}\right)=\frac{1}{2 i \pi} \int_{\Gamma} A(\zeta) f(\zeta) \frac{d \zeta}{\zeta-\zeta_{0}}+\sum_{n=1}^{\ell} A_{n}\left(\zeta_{n}\right) f\left(\zeta_{n}\right) \cdot\left(\zeta_{0}-\zeta_{n}\right)^{-1}
$$

where we denote $\Gamma=\{\zeta \in \mathbb{C} ;|\zeta|=R\}$. For $\zeta \in \Gamma$, we get

$$
|A(\zeta)| \leq \prod_{n=1}^{\ell} \frac{\left|R-\zeta_{n}\right|}{R-\left|\zeta_{n}\right|}
$$

then the required statement appears.

Lemma 6. Let $f(z)$ be an analytic function on a disk $|z| \leq R$ in $\mathbb{C}$. Let $\zeta_{0}, \ldots, \zeta_{\ell}$ be pairwise distinct points in $|z|<R$. Then

$$
\left|f\left(\zeta_{0}\right)\right| \leq G_{1}+G_{2}
$$

where

$$
G_{1}=|f|_{R} \cdot \frac{R}{R-\left|\zeta_{0}\right|} \prod_{n=1}^{\ell} \frac{R\left|\zeta_{0}-\zeta_{n}\right|}{\left|R^{2}-\zeta_{0} \bar{\zeta}_{n}\right|}
$$

and

$$
G_{2}=\sum_{n=1}^{\ell}\left(\left|f\left(\zeta_{n}\right)\right| \cdot \prod_{i=1}^{\ell}\left|\frac{R^{2}-\zeta_{n} \bar{\zeta}_{i}}{R^{2}-\zeta_{0} \bar{\zeta}_{i}}\right| \cdot \prod_{\substack{k=1 \\ k \neq n}}^{\ell}\left|\frac{\zeta_{0}-\zeta_{k}}{\zeta_{n}-\zeta_{k}}\right|\right)
$$

Proof. Write Blaschke product by 


$$
B(z)=\prod_{n=1}^{\ell} \frac{R^{2}-z \overline{\zeta_{n}}}{R\left(z-\zeta_{n}\right)}
$$

For $1 \leq n \leq \ell$ we also put

$$
B_{n}(z)=B(z)\left(z-\zeta_{n}\right)=\prod_{i=1}^{\ell} \frac{R^{2}-z \bar{\zeta}_{i}}{R} \prod_{\substack{k=1 \\ k \neq n}}^{\ell} \frac{1}{z-\zeta_{k}} .
$$

Again by the residue formula, we have

$$
B\left(\zeta_{0}\right) f\left(\zeta_{0}\right)=\frac{1}{2 i \pi} \int_{\Gamma} B(\zeta) f(\zeta) \frac{d \zeta}{\zeta-\zeta_{0}}+\sum_{n=1}^{\ell} B_{n}\left(\zeta_{n}\right) f\left(\zeta_{n}\right) \cdot\left(\zeta_{0}-\zeta_{n}\right)^{-1}
$$

where $\Gamma=\{\zeta \in \mathbb{C} ;|\zeta|=R\}$.

For $\zeta \in \Gamma$, we have $|B(\zeta)|=1$, then the proof of the lemma is achieved.

\section{Proof of Theorem 1}

Let $N_{0}$ be a sufficiently large integer. Let $h_{0} \in \mathbb{Q}$ such that $h_{0} N_{0} \in \mathbb{Z}, 0<h_{0}<\frac{1}{2}$. Let $k_{0} \in \mathbb{Z}$ with $k_{0} \geq 1$. Let $g>0$ such that

$$
\|f(n)\|<e^{-g n} .
$$

Finally, let $\lambda>1$ be a parameter $\in \mathbb{R}$.

[First step]: construction of an auxiliary function.

Write

$$
f_{j}(z)=\left(\begin{array}{l}
z \\
h
\end{array}\right) f(z)^{k}
$$

for $1 \leq h \leq h_{0} N_{0}, 0 \leq k \leq k_{0}-1,1 \leq j=h(k+1) \leq L=h_{0} k_{0} N_{0} \in \mathbb{Z}$.

Suppose that the entire function $f(z)$ satisfies for sufficiently large $r>0$ :

$$
\log |f|_{r}<\alpha r \text {. }
$$

Let $b_{n} \in \mathbb{Z}$ such that $\|f(n)\|=\left|f(n)-b_{n}\right| \leq e^{-g n}$ for all sufficiently large $n$ and put

$$
a_{j n}=\left(\begin{array}{l}
n \\
h
\end{array}\right) b_{n}^{k}
$$

for $1 \leq h \leq h_{0} N_{0}, 0 \leq k \leq k_{0}-1,1 \leq j=h(k+1) \leq L=h_{0} k_{0} N_{0}$.

We construct an auxiliary function

$$
F(z)=\sum_{1 \leq j \leq L} p_{j} f_{j}(z)
$$

with $p_{j} \in \mathbb{Z}$ not all zero such that

$$
\sum_{1 \leq j \leq L} p_{j} a_{j n}=0, \quad u\left(N_{0}-1\right) \leq n \leq N_{0}-1
$$

with a real parameter $u$ satisfying $0<u<1$.

By definition of $a_{j n}$ and our assumption on the growth of $f(z)$, if $h_{0}<\min (u, 1 / 2)$, then we have

$$
\log \max _{1 \leq j \leq L}\left|a_{j n}\right| \leq c_{1} N_{0}+\mathrm{o}\left(N_{0}\right)
$$

where

$$
c_{1}=\alpha\left(k_{0}-1\right)-\varphi\left(h_{0}\right)-\varphi\left(1-h_{0}\right) .
$$

Suppose $h_{0} k_{0}>1-u$. Then by Siegel's lemma (confer for example [11]) there exist integers $p_{j}, 1 \leq j \leq L$ which are not all zero such that

$$
\max _{1 \leq j \leq L} \log \left|p_{j}\right| \leq \frac{1-u}{h_{0} k_{0}+u-1} \times c_{1} N_{0}+\mathrm{o}\left(N_{0}\right) .
$$

For $N \geq N_{0}$ and $\lambda>1$, it follows

$$
\log |F|_{\lambda N} \leq\left(\frac{1-u}{h_{0} k_{0}+u-1} \times c_{1}+c_{2}\right) N+\mathrm{o}(N)+\mathrm{o}\left(N_{0}\right)
$$


with

$$
c_{2}=\varphi\left(\lambda+h_{0}\right)-\varphi(\lambda)-\varphi\left(h_{0}\right)+\alpha \lambda\left(k_{0}-1\right) .
$$

[Second step]: extrapolation.

We consider for each $N \geq N_{0}$ the following two properties:

$$
\begin{array}{ll}
A(N): & \sum_{1 \leq j \leq L} p_{j} a_{j n}=0, \quad u(N-1) \leq n \leq N-1, \\
B(N): \quad \log |F|_{N}<-\gamma N,
\end{array}
$$

where $\gamma>0$ is independent of $N_{0}$ and $N$.

We shall show $A(N) \Longrightarrow B(N)$ and $B(N) \Longrightarrow A(N+1)$.

Proof of $A(N) \Longrightarrow B(N)$. For $u(N-1) \leq n \leq N-1$ and $0 \leq k \leq k_{0}-1$, we have:

$$
\begin{aligned}
\max _{1 \leq j \leq L} \log \left|f_{j}(n)-a_{j n}\right| & \leq \max _{1 \leq j \leq L} \log \left|\left(\begin{array}{l}
n \\
h
\end{array}\right)\left(f(n)^{k}-b_{n}^{k}\right)\right| \\
& \leq c_{1} N-\alpha N-g u N+\mathrm{o}(N) .
\end{aligned}
$$

By hypothesis $A(N)$, it follows

$$
\log \sum_{u(N-1) \leq n \leq N-1}|F(n)| \leq \frac{h_{0} k_{0}}{h_{0} k_{0}+u-1} c_{1} \times N-(\alpha+g u) N+\mathrm{o}(N)+\mathrm{o}\left(N_{0}\right)
$$

By Lemma 6, we have

$$
|F|_{N} \leq T_{1}+T_{2}
$$

with

$$
T_{1}=|F|_{\lambda N} \frac{\lambda}{\lambda-1} \prod_{n} \frac{\lambda N(N+n)}{\lambda^{2} N^{2}+N n}
$$

and

$$
T_{2}=\left(\sum_{n}|F(n)|\right) \frac{1}{\left(\Gamma\left(\frac{1-u}{2} N-1\right)\right)^{2}} \prod_{n} \frac{\left(\lambda^{2} N^{2}-u(N-1) n\right)(N+n)}{\lambda^{2} N^{2}+N n}
$$

because $(z-n)\left(\lambda^{2} N^{2}-z n\right)^{-1}$ takes the maximal absolute value on the disk $\{|z|=N\}$ at the point $z=-N$. Therefore we obtain

$$
\begin{aligned}
\log T_{1} & \leq \log |F|_{\lambda N}+N(1-u) \log \lambda+\log \frac{\Gamma(2 N) \cdot \Gamma\left(\left(\lambda^{2}+u\right) N\right)}{\Gamma((1+u) N) \cdot \Gamma\left(\left(\lambda^{2}+1\right) N\right)}+\mathrm{o}(N)+\mathrm{o}\left(N_{0}\right) \\
& \leq\left(\frac{1-u}{h_{0} k_{0}+u-1} \times c_{1}+c_{2}+c_{3}\right) N+\mathrm{o}(N)+\mathrm{o}\left(N_{0}\right)
\end{aligned}
$$

where

$$
c_{3}=(1-u) \log \lambda+\varphi(2)+\varphi\left(\lambda^{2}+u\right)-\varphi(1+u)-\varphi\left(\lambda^{2}+1\right)
$$

and also

$$
\begin{aligned}
\log T_{2} \leq & \log \left(\sum_{n}|F(n)|\right)+\log \frac{\Gamma\left(\left(\frac{\lambda^{2}}{u}-u\right) N\right) \cdot \Gamma\left(\left(\lambda^{2}+u\right) N\right) \cdot \Gamma(2 N)}{\Gamma\left(\left(\frac{\lambda^{2}}{u}-1\right) N\right) \cdot \Gamma\left(\left(\lambda^{2}+1\right) N\right) \cdot \Gamma((1+u) N) \cdot\left(\Gamma\left(\frac{1-u}{2} N\right)\right)^{2}} \\
& +N(1-u) \log u+\mathrm{o}(N)+\mathrm{o}\left(N_{0}\right) \\
\leq & \left(\frac{h_{0} k_{0}}{h_{0} k_{0}+u-1} \times c_{1}-(\alpha+g u)+c_{4}\right) N+\mathrm{o}\left(N_{0}\right)+\mathrm{o}(N)
\end{aligned}
$$

where

$$
c_{4}=\varphi\left(\frac{\lambda^{2}}{u}-u\right)+\varphi\left(\lambda^{2}+u\right)+\varphi(2)-\varphi\left(\frac{\lambda^{2}}{2}-1\right)-\varphi\left(\lambda^{2}+1\right)-\varphi(1+u)-2 \varphi\left(\frac{1-u}{2}\right)+(1-u) \log u .
$$

Hence the property $B(N)$ is true for $N_{0}$ sufficiently large, whenever the following inequalities are satisfied:

$$
\frac{1-u}{h_{0} k_{0}+u-1} \times c_{1}+c_{2}+c_{3}<0
$$

and 


$$
\frac{h_{0} k_{0}}{h_{0} k_{0}+u-1} \times c_{1}-(\alpha+g u)+c_{4}<0
$$

Proof of $B(N) \Longrightarrow A(N+1)$. We are going to show that the property $B(N)$ implies $A(N+1)$.

We apply Liouville's theorem. Obviously:

$$
\left|\sum_{j} p_{j} a_{j N}\right| \leq\left|\sum_{j} p_{j}\left(a_{j N}-f_{j}(N)\right)\right|+|F(N)|
$$

where by assumption $B(N)$ we have,

$$
\log |F(N)| \leq \log |F|_{N}<-\gamma N
$$

On the other hand, Lemma 3 shows

$$
\log \left|\sum_{j} p_{j}\left(a_{j N}-f_{j}(N)\right)\right| \leq\left(\frac{1-u}{h_{0} k_{0}+u-1} \times c_{1}-\varphi\left(h_{0}\right)-\varphi\left(1-h_{0}\right)+\left(k_{0}-2\right) \alpha-g\right) N+\mathrm{o}(N)+\mathrm{o}\left(N_{0}\right) .
$$

Then the property $A(N)$ is satisfied when $N_{0}$ is sufficiently large and whenever the following condition holds:

$$
\frac{1-u}{h_{0} k_{0}+u-1} \times c_{1}-\varphi\left(h_{0}\right)-\varphi\left(1-h_{0}\right)+\left(k_{0}-2\right) \alpha-g<0 .
$$

Carrying out technical computational optimizations, we choose parameters such that the three conditions (6), (7), and (8) are simultaneously satisfied.

We set:

$$
h_{0}=0.108496, k_{0}=16, u=0.108496, \lambda=11.9194, g=663143,
$$

then the inequalities (6), (7), and (8) are verified with

$$
\alpha=0.00437155\left(>\frac{1}{229}\right)
$$

\section{[Third step]: conclusion.}

By the construction of the auxiliary function $F(z)$, the property $A\left(N_{0}\right)$ is true. Since $A(N) \Longrightarrow B(N)$ and $B(N) \Longrightarrow A(N+1)$, both properties $A(N)$ and $B(N)$ hold for any $N \geq N_{0}$. If the function $F(z)$ is not identically zero, then by Liouville's theorem, we obtain for $N \rightarrow \infty$ :

$$
|F|_{N} \rightarrow \infty
$$

which contradicts $B(N)$.

As a consequence, the function $F(z)$ is identically zero, namely the functions $f_{j}(z)$ are linearly dependent over $\mathbb{Q}$. Since it is equivalent to say that $f(z)$ is a polynomial with coefficients in $\mathbb{Q}$ and that the functions $f_{j}(z)=\left(\begin{array}{l}z \\ h\end{array}\right) f(z)^{k}(h, k \in$ $\mathbb{N} \cup\{0\}$ ) are linearly dependent over $\mathbb{Q}$, hence the proof of Theorem 1 is achieved.

\section{Proof of Theorem 2}

Take

$$
h_{0}=0.000991044, k_{0}=640, u=0.78256, \lambda=1.5253, g=3 \text {, }
$$

then the conditions (6), (7), and (8) are valid with

$$
\alpha=8.775 \times 10^{-6}
$$

which establishes Theorem 2.

\section{REFERENCES}

[1] Beukers, F., and Wolfart, J., Algebraic Values of Hypergeometric Functions, In New advances in Transcendence Theory (ed. A. Baker), Cambridge Univ. Press (1988) 68-81.

[2] Gramain, F., "Sur le théorème de Fukasawa-Gel'fond," Invent. Math., 63: 495-506 (1981).

[3] Gramain, F., Mignotte, M., and Waldschmidt, M., "Valeurs algébrique de fonctions analytiques," Acta Arith., 47: 97-121 (1986).

[4] Hardy, G. H., "On a theorem of G. Pólya," Proc. Cambridge Philosophical Society, 19: 60-63 (1916-1919).

[5] Hirata, N., "Depéndance linéaire de fonctions arithmétiques et presque arithmétiques," Proc. of the Japan Academy, Series A, 61 [No. 8]: 274-277 (1985). 
[6] Philippon, P., and Waldschmidt, M., "Formes linéaires de logarithmes sur les groupes algébriques commutatifs," Illinois $J$. Math., 32: 281-314 (1988).

[7] Pisot, Ch., "Sur les fonctions analytiques arithmétiques et presque arithmétiques," C. R. Acad. Sci. Paris, 222: 1027-1028 (1946).

[8] Pólya, G., "Über ganzwertige ganze Funktionen,” Rend. Circ. Math. Palermo, 40: 1-16 (1915).

[9] Stäckel, P., "Über arithmetische Eigenschaften analytischer Functionen,” Math. Annalen, 46: 513-520 (1895).

[10] Waldschmidt, M., "Pólya's theorem by Schneider's method," Acta Math. Acad. Sci. Hungar., 31: 21-25 (1978).

[11] Waldschmidt, M., Diophantine Approximation on Linear Algebraic Groups, Grundlehren der Math. 326, Wissenschaften, Springer (2000).

[12] Whittaker, E. T., and Watson, G. N., A Course of Modern Analysis, Cambridge Math. Library, the fourth edition, Cambridge Univ. Press (1927). 